\title{
Power Adjustment and Scheduling in OFDMA Femtocell Networks
}

\author{
Michael Lin \\ CSE Department \\ Pennsylvania State University \\ Email:molin@cse.psu.edu
}

\author{
Novella Bartolini \\ Computer Science Department \\ Sapienza University, Italy \\ Email:novella@di.uniroma1.it
}

\author{
Thomas La Porta \\ CSE Department \\ Pennsylvania State University \\ Email:tlp@cse.psu.edu
}

\begin{abstract}
Densely-deployed femtocell networks are used to enhance wireless coverage in public spaces like office buildings, subways, and academic buildings. These networks can increase throughput for users, but edge users can suffer from co-channel interference, leading to service outages. This paper introduces a distributed algorithm for network configuration, called Radius Reduction and Scheduling (RRS), to improve the performance and fairness of the network. RRS determines cell sizes using a Voronoi-Laguerre framework, then schedules users using a scheduling algorithm that includes vacancy requests to increase fairness in dense femtocell networks. We prove that our algorithm always terminate in a finite time, producing a configuration that guarantees user or area coverage. Simulation results show a decrease in outage probability of up to $50 \%$, as well as an increase in Jain's fairness index of almost $200 \%$.
\end{abstract}

\section{INTRODUCTION}

Femtocells are small, locally-deployed wireless base stations that have become popular as a means of supporting or replacing existing wireless macrocells. Their key features are: a small coverage area, on the order of 100 square meters; automatic setup and the use of the customer's Internet connection for backhaul, which allow them to be deployed by customers rather than service providers; customer-defined access control.

Densely deployed femtocells can be used to establish wireless networks that operate in a similar manner to public or enterprise WiFi networks, but serve users using $3 \mathrm{G}$ or $4 \mathrm{G}$ wireless technology. These types of networks face different challenges than both WiFi networks and independently deployed femtocells. The load on such networks is typically much higher than on isolated femtocells, and co-channel interference between femtocells can cause service degradation. Due to these issues, recently dense femtocell networks have been an active topic in the research community. For instance, Arslan, et al. [1] introduce FERMI, a system for interference mitigation in dense OFDMA femtocell networks, that uses concepts from fractional frequency reuse to mitigate the negative performance impact of interference. Wang, et al. [2] present a joint power-and-fairness optimization algorithm for dense femtocell networks.

Due to the network environment, edge users in dense femtocell networks can be exposed to high interference, leading to reduced throughput and fairness. Fairness in wireless networks refers to the ability of the network to share its resources amongst all users, rather than using its resources to serve only the users with the best signal quality, which maximizes system throughput but can starve some users. Fairness has typically been approached as a scheduling or resource management [3], [4], [5], [6], [7] problem. See [8] for a comprehensive discussion of fairness in wireless networks.

This paper takes a different approach, and introduces a new algorithm, RAdIUS REDUCTION AND SCHEDULING (RRS), that increases fairness in densely deployed femtocell networks using a combination of power management and resource block scheduling.

Given the locations of users and femtocells, RRS determines the network configuration in two phases. In the first phase, it uses a Voronoi-Laguerre geometry-derived framework to reduce femtocell coverage overlaps and co-channel interference. At the same time, this preliminary phase seeks to maximize the number of users that can be served within their quality of service requirements. The first phase of the algorithm provides an iterative adjustment of each femtocell radius, on the basis of only local information on the setting of neighbor cells. We prove the termination of this phase in a finite number of steps, and we show that it preserves either area or user coverage while reducing transmission power and femtocell radio coverage overlaps and interference. In the second phase, RRS provides a resource block scheduling scheme that uses vacancy requests to improve resource sharing and service of users that are unable to meet their performance targets.

Simulations of an LTE-based network are used to compare RRS to previous approaches. We show that RRS can reduce the outage probability, the percentage of users that cannot meet their throughput requirements, by up to $100 \%$ over a baseline algorithm working with fixed cell radii and best-effort scheduling. Furthermore, our algorithm increases the Jain's index [9] of the network, a common measure of fairness, by up to $190 \%$.

The contributions of this paper can be summarized as follows:

- We propose a new algorithm, called RRS, for reducing femtocell coverage radii and scheduling resource blocks among users. This algorithm can be formulated in several variants to consider different performance objectives

- We prove that RRS terminates in a finite time and preserves either area or user coverage, despite radius 


\begin{tabular}{|l|l|}
\hline Notation & Description \\
\hline$F$ & set of femtocells \\
\hline$f_{i} \in F$ & i-th femtocell \\
\hline $\mathcal{U}$ & set of users \\
\hline$u_{j} \in \mathcal{U}$ & $j$-th user \\
\hline $\mathcal{P}$ & possible power values of a femtocell \\
\hline$\pi_{i} \in \mathcal{P}$ & power level of $f_{i}$ \\
\hline$B^{\mathrm{Max}}$ & number of resource blocks for any femtocell \\
\hline$\varsigma_{j}$ & estimated SINR of user $u_{j}$ \\
\hline$t_{j}$ & instantaneous throughput of user $u_{j}$ \\
\hline$\tau_{j}$ & throughput requirement of user $u_{j}$ \\
\hline$V\left(\mathcal{C}_{i}\right)$ & Laguerre polygon of circular range $\mathcal{C}_{i}$ \\
\hline$V\left(f_{i}\right)$ & Laguerre polygon of femtocell $f_{i}$ \\
\hline$V^{(k)}\left(f_{i}\right)$ & polygon $V\left(f_{i}\right)$ at iteration $k$ \\
\hline$\hat{U}_{i}^{(k)}$ & farthest user of $V^{(k)}\left(f_{i}\right)$ \\
\hline$\hat{V}_{i}^{(k)}$ & farthest vertex of $V^{(k)}\left(f_{i}\right)$ \\
\hline$r_{i}^{(k)}$ & radius of femtocell $f_{i}$ at iteration $k$ \\
\hline $\mathcal{S}^{(k)}$ & area covered at iteration $k$ \\
\hline $\mathcal{U}^{(k)}$ & users covered at iteration $k$ \\
\hline $\mathcal{U}^{(k)}\left(f_{i}\right)$ & set of users inside $V^{(k)}\left(f_{i}\right)$ \\
\hline$\alpha_{i}^{(k)}$ & radius reduction rate of $f_{i}$ at iteration $k$ \\
\hline$\epsilon_{\alpha}$ & minimum reduction rate \\
\hline
\end{tabular}

TABLE I: Summary of notations

reduction

- We provide simulation results from a dynamic, interfering OFDMA system simulator showing that our algorithm reduces outage probabilities and increases fairness.

\section{Network Model and Problem Formulation}

In this section we define the femtocell network model and introduce our assumptions and notation. Table I summarizes the notation used throughout the paper. We consider an OFDMA network with several femtocells $f_{i} \in F$, interfering with each other. Femtocell $f_{i}$ may adjust its power $\pi_{i}$ within a range $\mathcal{P}$ of possible values. We consider a set of users $\mathcal{U}$, and we assume that femtocells are deployed densely enough to cover all users of $\mathcal{U}$ when they work at maximum power. We assume that users preferentially associate with femtocells. Nevertheless, in order to have a realistic model, we also consider the presence of a macrocell. Thanks to the joint presence of both the macrocell and the femtocells in our model, we are able to capture the interference generated by the macrocell.

A femtocell's available bandwidth is time- and frequencydivided into resource blocks (RB), with $B^{\mathrm{Max}}$ numbered resource blocks per femtocell, as in an LTE system.

Resource blocks deliver a varying number of bits, depending on the signal-to-interference-and-noise ratio (SINR) received by a user on that resource block, and on the subsequent channel quality indicator (CQI) measured on the resource block itself. CQIs are a measurement of the channel quality between a user and its serving base station. CQIs are determined by a step-wise function defined on the SINR of an individual resource block.

The estimated SINR $\varsigma_{j}$ of a user $u_{j}$ is measured using reference signals. Notice that, since OFDMA signals are spread across a wide spectrum range, individual subchannel SINRs may differ significantly from $\varsigma_{j}$. The instantaneous throughput $t_{j}$ of user $u_{j}$ is the sum of the throughput achieved on each

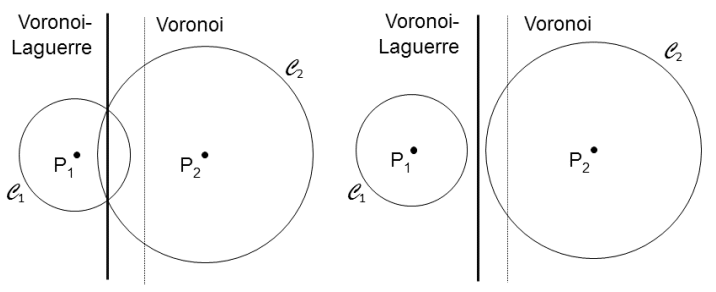

Fig. 1: Voronoi and Voronoi-Laguerre cell boundaries

resource block assigned to the user during a transmission frame.

We tackle the problem of increasing the fairness of a wireless network, with the ancillary goal of decreasing power consumption. We consider users having heterogeneous requirements. We define a hard throughput requirement $\tau_{j}$ for each user $u_{j}$, used to determine whether a user is served or not. Our goal is to serve the maximum number of users under this constraint, at the possible expense of reduced global throughput.

We address this problem by means of a new algorithm, Radius REDUCTION AND SCHEDUling (RRS) that determines power and resource block scheduling, given femtocells and users positions. The algorithm RRS runs in all femtocells across the network at network initialization, and in local femtocells when users arrive or move between femtocells. It works in two phases.

The first phase of RRS determines the femtocell transmission power. Each femtocell configures its own transmission power on the basis of a distributed coordination protocol that allows neighboring femtocells (femtocells that are in radio proximity with each other) to exchange information and adapt their working setting cooperatively. By using this distributed coordination protocol, neighboring femtocells determine their respective responsibility regions, namely the regions where each cell is responsible for providing enough radio resources to serve users.

In the second phase of RRS the algorithm provides a resource block scheduling scheme, so that users working on conflicting radio resources are able to share them and to perform non conflicting transmissions.

In Sections IV and V we describe the two phases of radius reduction and scheduling, respectively. In the next Section, we introduce the mathematical background of Laguerre geometry to motivate the design of the radius reduction phase.

\section{BACKGROUND ON VORONOI-LAGUERRE DIAGRAMS}

In typical wireless networks, users associate with the base station that has the strongest reference signal. Under the assumption of homogeneous transmission power, the coverage cells resulting from this type of user association can be approximated using Voronoi cells, where $f_{i}$ 's Voronoi cell consists of all points closer to $f_{i}$ than to any other femtocell. The Voronoi cell approximation has been extensively studied and shown to be good under certain wireless network scenarios [10]. 
In a Voronoi diagram, cell polygons are defined by the axis of the segments generated by two femtocells, that is the locus of the points that are equidistant from them and perpendicular to their connecting segment (Voronoi line in Figure 1). Given any two femtocells, this line divides the plane into two halves. If the femtocell transmission power is homogeneous, the two femtocells would have the same coverage radius, and the Voronoi line would properly delimit their responsibility regions. Nevertheless, when the cells have different radio coverage capabilities, the Voronoi line may not determine the responsibility region correctly, as shown in Figure 1.

In both the diagrams the Voronoi cell assigns some points that are better served by the femtocell on the right, located at $P_{2}$, to the femtocell on the left, at $P_{1}$. The desired partition of the plane into responsibility regions is through the intersection of the circles representing the radio coverage range of the two cells, labelled the Voronoi-Laguerre line in Figure 1. This line is perpendicular to the segment connecting the positions $P_{1}$ and $P_{2}$ of the two femtocells, and equidistant in the Laguerre geometry. Voronoi-Laguerre cells are defined using the Laguerre distance, $d_{L}^{2}$, which defines a radius-dependent distance between two circles, or between a circle and a point. Consider the circles $\mathcal{C}_{1}$ and $\mathcal{C}_{2}$, with respective radii $r_{1}$ and $r_{2}$, with $r_{2}>r_{1}$, in Figure 1. The boundary between the Voronoi cells of $\mathcal{C}_{1}$ and $\mathcal{C}_{2}$ is indicated by the dashed line, while the boundary between the Voronoi-Laguerre cells is indicated by the solid line.

The Laguerre distance between two circles $\mathcal{C}_{1}$ and $\mathcal{C}_{2}$ centered on points $P_{1}$ and $P_{2}$, with radii $r_{1}$ and $r_{2}$, respectively, is defined as: $d_{L}^{2}\left(\mathcal{C}_{1}, \mathcal{C}_{2}\right)=\left\|p_{1}-p_{2}\right\|^{2}-\left(r_{1}-r_{2}\right)^{2}$. This definition can also be used to calculate the distance between a circle and a point, by considering the point as a circle with null radius. It is straightforward to see that under the Laguerre geometry, given two circles with distinct centers and possibly different radii, the locus of the points equally distant from them is a line, hereby called the Voronoi-Laguerre line, that is perpendicular to the segment connecting the centers, with the following properties: if the two circles intersect each other, their Voronoi-Laguerre line crosses their intersection points, as in the left side of Figure 1, while if two circles are disjoint, the Voronoi-Laguerre line lies between them, as in the diagram on the right of Figure 1.

Notice also that this definition implies that, depending on the overlap between two circles, in the Laguerre geometry the two centers may fall on the same side of the Voronoi Laguerre line, which would imply that the responsibility region of one femtocell would be located on the opposite side of the Voronoi Laguerre line with respect to its center [11]. The notion of Voronoi diagrams can be extended to the Laguerre geometry, as follows: given $N$ circles $\mathcal{C}_{i}$ with centers $\mathrm{C}_{i}=\left(x_{i}, y_{i}\right)$ and radii $r_{i}, i=1, \ldots, N$, the Voronoi-Laguerre polygons $V\left(\mathcal{C}_{i}\right)$ of the circles $\mathcal{C}_{i}$ are defined as

$$
V\left(\mathcal{C}_{i}\right)=\left\{\mathrm{P} \in \mathbb{R}^{2} \mid d_{L}^{2}\left(\mathcal{C}_{i}, \mathrm{P}\right) \leq d_{L}^{2}\left(\mathcal{C}_{j}, \mathrm{P}\right), j \neq i\right\} .
$$

Notice, that unlike with traditional Voronoi diagrams, this extension to the Laguerre geometry may lead to the case that some polygons are either null or empty [11], reflecting a situation of high coverage redundancy that is not captured by traditional Voronoi diagrams. Null and empty polygons are described in more detail in Section IV.

Voronoi-Laguerre diagrams are extraordinarily powerful in modeling the responsibility regions of heterogeneous femtocells. Indeed, a fundamental property of the Voronoi diagrams in Laguerre geometry is the following:

Theorem III.1. ([12]) Let us consider $N$ circles $\mathcal{C}_{i}$, with centers $C_{i}=\left(x_{i}, y_{i}\right)$ and radii $r_{i}, i=1, \ldots, N$, and let $V\left(\mathcal{C}_{i}\right)$ be the Voronoi-Laguerre polygon of the circle $\mathcal{C}_{i}$. For all $k, j=1,2, \ldots, N, V\left(\mathcal{C}_{k}\right) \cap \mathcal{C}_{j} \subseteq \mathcal{C}_{k}$

Less formally, if a point $P$ is in the coverage range of at least one femtocell, it is certainly covered also by the femtocell $f_{i}$ that generates the Voronoi-Laguerre polygon $V\left(\mathcal{C}_{i}\right)$ that includes $P$.

In the following, we refer to $V\left(f_{i}\right)$ as to the VoronoiLaguerre polygon of femtocell $f_{i}$, or alternatively, the responsibility region of cell $f_{i}$. Likewise, we denote with $V^{(k)}\left(f_{i}\right)$ the Voronoi Laguerre polygon of femtocell $f_{i}$ at iteration $k$.

\section{RADIUS REDUCTION}

This section describes the radius reduction phase of RSS. First, we prove that our coverage criteria preserve either user or area coverage, then we describe their use in the algorithm in detail. After that, we prove that the algorithm terminates, and discuss the reduction rate parameter, $\alpha$.

The goal of this phase is to obtain a network configuration with less overlap in the radio coverage of neighboring femtocells, so as to limit co-channel interference which would reduce radio resource availability. This is done by eliminating unncessary femtocells and reducing the transmission power of the remaining femtocells. This phase is executed in a distributed manner and governs the reduction of a femtocell's transmit power and consequently, its radio coverage range. Since the range reduction is performed in a distributed manner, it is necessary to design an algorithm that allows neighboring cells to coordinate with each other, so as to avoid conflicting decisions that may lead to a loss of service in some regions.

Each femtocell calculates its transmission power iteratively. The algorithm begins with all femtocells transmitting at maximum power. At any iteration $k$, each femtocell $f_{i}$ reduces its transmission power $p_{i}^{(k)}$ and its transmission radius $r_{i}^{(k)} \triangleq$ $r_{i}\left(p^{(k)}\right)$ correspondingly. It must be noted that since every cell potentially reduces its radius by some amount, the VoronoiLaguerre diagram determining the responsibility regions of each cell is recalculated locally at any iteration $k$ until the algorithm converges.

The amount of radius reduction at each iteration is determined according to one of two criteria:

- Radius reduction preserving user coverage (UCR) Each femtocell reduces its radius ensuring that it does not leave uncovered any of the users residing in its responsibility region at the current iteration. It does so by limiting the 
amount of reduction so as to preserve coverage of the farthest user located in its polygon.

- Radius reduction preserving area coverage (ACR) Each femtocell reduces its radius ensuring that it does not reduce the coverage of its current iteration VoronoiLaguerre polygon. It does so by limiting the amount of reduction so as to preserve radio coverage of the farthest point of its polygon.

The ACR approach is inspired to the works of Gupta et al. [13] and Bartolini et al. [14] that are designed for selective activation and radius adaptation of sensor networks to provide sensing coverage, and aim at preserving coverage completeness of a continuous area of interest. By contrast, UCR is meant to ensure radio coverage where it is actually needed, that is in the discrete points of the area where users are located. Note that UCR does not aim to ensure completeness of area coverage.

\section{A. Preservation of Coverage}

In this subsection, we establish our criteria for eliminating femtocells while maintaining either user or area coverage. Let us consider a femtocell $f_{i}$, located at $P_{i}$. Let $\hat{U}_{i}^{(k)}$ be the position of the farthest covered user lying in the VoronoiLaguerre polygon of $f_{i}$, and let $\hat{V}_{i}^{(k)}$ be the farthest covered point of the same polygon at the current iteration $k$.

According to the UCR criterion, $f_{i}$ can reduce its radius for the $(k+1)$-th iteration to a value $r_{i}^{(k+1)} \leq r_{i}^{(k)}$ such that it still covers the position of the farthest user of its polygon:

$$
r_{i}^{(k+1)} \geq\left\|P_{i}-\hat{U}_{i}^{(k)}\right\|
$$

Similarly, according to the ACR criterion the next iteration value of $r_{i}^{(k+1)}$ must meet the area coverage constraint:

$$
r_{i}^{(k+1)} \geq\left\|P_{i}-\hat{V}_{i}^{(k)}\right\|
$$

Note that in the description of these two criteria for radius reduction we only considered non-empty and non-null polygons. An empty polygon is a Voronoi-Laguerre polygon that does not contain its generating point, that is the polygon $V\left(f_{i}\right)$ for which the position $P_{i}$ of the femtocell $f_{i}$ is such that $P_{i} \notin V\left(f_{i}\right)$. A null polygon is a degenerate polygon, with no points, that is $V\left(f_{i}\right)=\emptyset[11]$.

The Corollaries 1 and 2 follow from Theorem III. 1 and characterize the redundancy of femtocell $f_{i}$ in the aforementioned situations.

Corollary 1 (User coverage redundancy). If femtocell $f_{i}$ has an empty polygon $V\left(f_{i}\right)$ and it does not cover any of the users contained in it, or it has a null polygon, then $f_{i}$ is redundant, that is, for any user $u$ with position $P_{u}$ covered by $f_{i}$, there is another femtocell $f_{j}$ that also covers $P_{u}$.

Proof. This corollary extends Corollary 3.1 of [14] to the case in which a femtocell $f_{i}$ may cover a part of its polygon but does not cover any user. We recall that the Voronoi-Laguerre diagram of a region $\mathcal{R}$, constitutes a partition of $\mathcal{R}$. Let $\mathcal{C}\left(f_{i}\right)$ be the radio range of $f_{i}$. Any user positioned in $P_{u} \in \mathcal{C}\left(f_{i}\right)$ does not belong to $V\left(f_{i}\right)$ because, by assumption, $f_{i}$ does not cover any user lying in its polygon. Hence the location of any user covered by $f_{i}$ must belong to the Voronoi cell of some other femtocell $f_{j}$. By Theorem III.1 and since $P_{u}$ is covered by assumption, we can conclude that the user location $P_{u}$ is also covered by femtocell $f_{j}$. As this is true for any user in $\mathcal{C}\left(f_{i}\right)$ we can conclude that $f_{i}$ is redundant.

In less formal words, user coverage redundancy captures the situation in which femtocell $f_{i}$ covers only users that are better served by other femtocells (cells that are closer according to the Laguerre distance), or the situation in which $f_{i}$ is located such that it is too far away to cover the uncovered users located in its polygon, even at maximum transmission power. Analogously, we can prove Corollary 2, which directly derives from Corollary 3.1 of [14]; we omit the proof for space.

Corollary 2 (Area coverage redundancy). If femtocell $f_{i}$ has an empty polygon $V\left(f_{i}\right)$ and it does not cover any point of $i t$, or it has a null polygon, then $f_{i}$ is redundant, that is, for any point $Z$ covered by $f_{i}$, there is another femtocell $f_{j}$ that covers $Z$.

Area coverage redundancy captures the situation in which a femtocell $f_{i}$ covers only points that are better covered by other femtocells (cells that are closer according to the Laguerre distance), or is located such that it cannot cover the points of its area of responsibility.

Corollaries 1 and 2 allow us to define two situations of eliminable redundancy in which a femtocell (the eliminable femtocell) can be immediately disabled. Note that these corollaries define only sufficient conditions for redundancy and some femtocells can be redundant without meeting the criteria in Corollaries 1 and 2. In these cases a gradual iterative reduction of femtocell radio coverage range is needed to find redundant femtocells, a process which addresses possible conflicts in the concurrent elimination of several potentially redundant femtocells.

The following theorems show that if all femtocells apply an iterative radius reduction under the limits posed by the constraints in Equation 1 for the UCR criterion and Equation 2 for the ACR criterion, user coverage and area coverage are respectively preserved and eliminable femtocells are turned off. We define a distributed execution of radius reduction according to the UCR or the ACR criterion as the following: every non eliminable femtocell reduces its radius under the constraints given by Equation 1 or Equation 2, and every eliminable femtocell is turned off.

Theorem IV.1 (User coverage preservation under UCR). Let us consider a set $F$ of femtocells, randomly spread over a region $\mathcal{R}$. Let us also consider a set $\mathcal{U}$ of users over the same region. Let $\mathcal{U}^{(k)} \subseteq \mathcal{U}$ be the subset of users that the femtocells of $F$ are able to cover when each femtocell $f_{i}$ works with radius $r_{i}^{(k)}$. Let $\mathcal{U}^{(k+1)}$ be the set of users covered by the same femtocells of $F$ after a distributed execution of a radius reduction according to the UCR criterion. Then 
$\mathcal{U}^{(k)}=\mathcal{U}^{(k+1)}$, so the radius reduction preserves coverage of the users within the region $\mathcal{R}$.

Proof. For simplicity of notation let us consider the set $\mathcal{U}$ as a finite set of points in $\mathcal{R}$, representing user positions. The Voronoi-Laguerre diagram determined by the positions and radii of the femtocells of $F$ at iteration $k$ creates a partition of the set of $\mathcal{U}^{(k)}$ as follows: $\mathcal{U}^{(k)}=\cup_{f_{i} \in F} \mathcal{U}^{(k)}\left(f_{i}\right)$, with $\mathcal{U}^{(k)}\left(f_{i}\right) \cap \mathcal{U}^{(k)}\left(f_{j}\right)=\emptyset$, for $i \neq j$, and where $\mathcal{U}^{(k)}\left(f_{i}\right)$ is the set of users inside $V^{(k)}\left(f_{i}\right)$. By altering the radii of the femtocells the Voronoi-Laguerre diagram is also altered, and consequently users that were in the polygon of a cell at iteration $k$ may find themselves in another cell at iteration $k+1$. Nevertheless, in order to prove the theorem we need to ensure that any user that was covered at iteration $k$ will still be covered by at least one of the available femtocells even after the radius reduction performed at iteration $k+1$. To this purpose, it is sufficient to prove that such a radius reduction preserves coverage of all the covered user positions of each polygon according to the partition determined by the diagram at the $k$-th iteration. Thanks to Theorem III.1 we know that the covered users of each polygon $V^{(k)}\left(f_{i}\right)$ are also covered by $f_{i}$ itself, and therefore we can write $\mathcal{U}^{(k)}$ in terms of the union of the covered sets of user positions of each polygon as follows: $\mathcal{U}^{(k)}=\bigcup_{f_{i} \in F} \mathcal{C}^{(k)}\left(f_{i}\right) \cap \mathcal{U}^{(k)}\left(f_{i}\right)$.

Each non eliminable cell performs a radius reduction that, according to constraint (1), preserves coverage of the users of its polygon.

Therefore, $\mathcal{U}^{(k)}\left(f_{i}\right) \cap \mathcal{C}^{(k+1)}\left(f_{i}\right)=\mathcal{U}^{(k)}\left(f_{i}\right) \cap \mathcal{C}^{(k)}\left(f_{i}\right)$. The same is trivially true also for eliminable femtocells as they do not have any covered user in their polygons at iteration $k$. This concludes the proof, as

$$
\begin{aligned}
\mathcal{U}^{(k+1)} & =\bigcup_{f_{i} \in F} \mathcal{C}^{(k+1)}\left(f_{i}\right) \cap \mathcal{U}^{(k)}\left(f_{i}\right)= \\
& =\bigcup_{f_{i} \in F} \mathcal{C}^{(k)}\left(f_{i}\right) \cap \mathcal{U}^{(k)}\left(f_{i}\right)=\mathcal{U}^{(k)} .
\end{aligned}
$$

The following Theorem also holds for the criterion ACR and can be proved using a similar argument.

Theorem IV.2 (Area coverage preservation under ACR). Let us consider a set $F$ of femtocells, randomly spread over a region $\mathcal{R}$. Let $\mathcal{S}^{(k)} \subseteq \mathcal{R}$ be the portion of $\mathcal{R}$ that the femtocells of $F$ are able to cover when each femtocell $f_{i}$ works with radius $r_{i}^{(k)}$. Let $\mathcal{S}^{(k+1)}$ be the area covered by the same femtocells of $F$ after a distributed execution of a radius reduction according to the ACR criterion. Then $\mathcal{S}^{(k)}=\mathcal{S}^{(k+1)}$, so the radius reduction preserves coverage of the region $\mathcal{R}$.

We underscore that even though the radius reduction preserves area coverage and user coverage, according to criteria ACR and UCR, respectively, the modification in the femtocell radii significantly alter the shape of the Voronoi-Laguerre polygons at any iteration. Consequently, users can be logically reassigned at any given iteration. We will show later in this section that the iterative radius reduction rapidly converges to the final setting of the femtocell radii.

Thanks to Theorems IV.1 and IV.2, we are able to guarantee that even if every femtocell performs a radius reduction to the minimum value provided by Equations 1 and 2, user and area coverage is preserved. Nevertheless the range of possible values for each femtocell radius can be exploited to prioritize the radius reduction of some femtocells over the others according to a given performance objective.

\section{B. Radius Reduction}

We propose that the radius reduction be performed gradually, at every iteration $k$, with only a partial reduction $\alpha_{i}^{(k)} \in[0,1]$ for every femtocell $f_{i}$, at each step, as we describe in Algorithm 1. We call the parameter $\alpha_{i}^{(k)}$ the radius reduction rate of femtocell $f_{i}$ at iteration $k$. The formulation of this parameter is described in detail in Section IV-D. It requires neighboring femtocells to exchange additional information regarding their current iteration setting.

Under this approach, femtocell $f_{i}$ starts working at maximum power $p_{i}^{(0)}=p_{i}^{\mathrm{Max}}$ at iteration $k=0$. Let $p_{\mathrm{min}}^{(k)}(i)_{\mathrm{UCR} \mid \mathrm{ACR}}$ be the minimum value of power that ensures that $f_{i}$ covers either the farthest covered user $\hat{U}_{i}^{(k)}$ (under the UCR criterion) or the farthest covered point $\hat{V}_{i}^{(k)}$ (under the ACR criterion) of $V^{(k)}\left(f_{i}\right)$.

This value can be expressed as follows: under the UCR criterion it is $p_{\mathrm{min}}^{(k)}(i)_{\mathrm{UCR}}=\min \left\{\pi: r(\pi) \geq\left\|P_{i}-\hat{U}_{i}^{(k)}\right\|, \pi \in\right.$ $\mathcal{P}\})$, while under the ACR criterion, $p_{\min }^{(k)}(i)_{\mathrm{ACR}}=\min \{\pi$ : $\left.\left.r(\pi) \geq\left\|P_{i}-\hat{V}_{i}^{(k)}\right\|, \pi \in \mathcal{P}\right\}\right)$. At any given iteration $k$, the transmission power of $f_{i}$ is reduced to $p_{i}^{k+1} \leq p_{i}^{k}$, with:

$$
p_{i}^{(k+1)}=p_{i}^{(k)}-\alpha_{i}^{k} \cdot\left(p_{i}^{(k)}-p_{\mathrm{min}}^{(k)}(i)_{\mathrm{UCR} \mid \mathrm{ACR}}\right) .
$$

According to Equation 3 the maximum reduction of power is obtained for $\alpha_{i}^{k}=1$. We consider a positive lower bound $\epsilon_{\alpha}$ to $\alpha_{i}^{k}$, such that $\epsilon_{\alpha}>0$ and $\epsilon_{\alpha}<<1$ to ensure that all the femtocells that can reduce their radius are actually able to do so, regardless of the behavior of their neighboring cells.

A preliminary exchange of information among neighboring femtocells is needed to let each cell know the position and current radius of its neighbor femtocells to calculate the current iteration Voronoi-Laguerre polygon, and the current value of the radius reduction rate $\alpha_{i}^{(k)}$, as indicated in line 4 of Algorithm 1.

In most cases femtocells only allow tuning of the transmission power within a discrete set of values. In such cases, the algorithm should take the minimum of these discrete values that exceeds the calculated value of $p_{i}^{(k+1)}$. For the sake of clarity and without loss of generality we neglect this aspect in the following.

When femtocells have overlapping coverage ranges, the radius reduction algorithm can lead some femtocells to reduce their transmission powers to zero. When this occurs, femtocells with zero transmission power serve no users, and do not transmit at all during the next frame, until a network reconfiguration, which may occur as a consequence of user movement, arrival, or departure.

The distributed radius reduction procedure is described in detail in Algorithm 1. This procedure guarantees that $f_{i}$ 's transmission power, $p_{i}^{(k)}$, is reduced to the minimum value of 


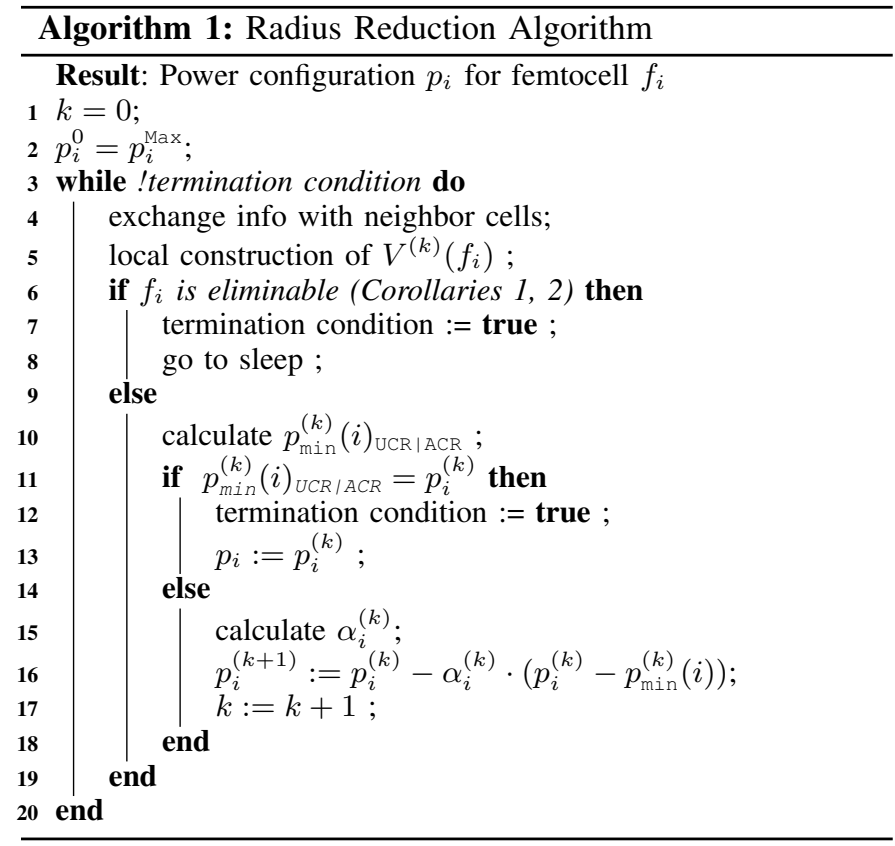

power that ensures either area or user coverage according to criterion ACR or UCR, respectively. The speed of $f_{i}$ 's radius reduction is determined by the parameter $\alpha_{i}^{(k)}$. Section IV-D is devoted to a discussion of possible ways to set the parameter $\alpha_{i}^{(k)}$.

\section{Termination of $R R S$}

The radius reduction algorithm provably terminates provided that the radius reduction at each step is a finite amount, which is always the case when radius reduction is limited to discrete steps.

Theorem IV.3 (Convergence of UCR). Given a set of $F$ femtocells with tunable radii, executing the radius configuration phase of the algorithm RRS under the UCR criterion, each femtocell converges [in a finite time] to a final radius configuration.

Proof. If $V^{(k)}\left(f_{i}\right)$ is not covered, $f_{i}$ is eliminable and goes to sleep. If $V^{(k)}\left(f_{i}\right)$ is at least partially covered, then $f_{i}$ can reduce its radius up to an extent that preserves coverage of the farthest covered user. As $\alpha_{i}^{(k)} \geq \epsilon_{(\alpha)}>0$, the algorithm performs a reduction at any iteration, until the radius becomes null or equals the distance to the farthest user covered exclusively. Termination follows by setting a finite and positive minimum value of radius reduction $\epsilon_{\mathrm{r}}$ that can be performed in a single iteration.

Theorem IV.4 (Convergence of ACR). Given a set of $F$ femtocells with tunable radii, executing the radius configuration phase of the algorithm RRS under the ACR criterion, each femtocell converges [in a finite time] to a final radius configuration.

Proof. The proof follows similarly to the proof of Theorem IV.3, with the only exception that if $V^{(k)}\left(f_{i}\right)$ is only partially covered, then $f_{i}$ cannot reduce its radius as it needs to preserve coverage of the farthest covered point. So the algorithm immediately terminates for those femtocells covering their polygons only partially.

\section{Radius Reduction Rate $\alpha_{i}$}

The radius reduction rate $\alpha_{i}$ controls the priority with which $f_{i}$ reduces its transmission power with respect to its neighbor femtocells. In the following we introduce three different prioritization criteria, that correspond to different ways to calculate $\alpha_{i}^{(k)}$. In all the approaches, only the femtocells with reduction rate $\alpha_{i}^{(k)}=1$ are allowed to perform the maximum allowed radius reduction.

1) Prioritization based on throughput increase (TI): Let $t^{(k)}\left(f_{i}\right)$ be the estimated increase in total throughput that would be achieved by femtocell $f_{i}$ if it decreases its transmission power to the minimum value.

We denote with $\mathcal{N}_{i}$ is the set of femtocells in radio proximity to $f_{i}$, and define $t_{\text {min }}^{(k)}\left(\mathcal{N}_{i}\right)$ and $t_{\text {Max }}^{(k)}\left(\mathcal{N}_{i}\right)$ the minimum and maximum value of the same metric in the neighbor cells of $f_{i}$. Namely, $t_{\text {min }}^{(k)}\left(\mathcal{N}_{i}\right) \triangleq \min _{f_{j} \in \mathcal{N}_{i}} t^{(k)}\left(f_{j}\right)$. Similarly, $t_{\text {Max }}^{(k)}\left(\mathcal{N}_{i}\right) \triangleq \max _{f_{j} \in \mathcal{N}_{i}} t^{(k)}\left(f_{j}\right)$. The value of $\alpha_{i}^{(k)}$ under the $T I$ criterion of prioritization is therefore the following:

$$
\alpha_{i}^{(k)}=\frac{t^{(k)}\left(f_{i}\right)-t_{\mathrm{min}}^{(k)}\left(\mathcal{N}_{i}\right)}{t_{\mathrm{Max}}^{(k)}\left(\mathcal{N}_{i}\right)-t_{\mathrm{min}}^{(k)}\left(\mathcal{N}_{i}\right)} .
$$

2) Prioritization based on number of users meeting throughput requirements (UTR): When the metric of interest in the prioritization is the number of users that achieve their quality requirements, we can denote with $u^{(k)}\left(f_{i}\right)$ the number of users that would be able to meet their throughput requirement if the radius of cell $f_{i}$ were reduced at a minimum. Similarly to what we did for the throughput increase criterion, we denote with $u_{\min }^{(k)}\left(\mathcal{N}_{i}\right)$ and $u_{\operatorname{Max}}^{(k)}\left(\mathcal{N}_{i}\right)$, the minimum and the maximum of the same metric over the neighbors of $f_{i}$. Under the UTR criterion of prioritization we define the value of $\alpha_{i}^{(k)}$ as follows:

$$
\alpha_{i}^{(k)}=\frac{u^{(k)}\left(f_{i}\right)-u_{\min }^{(k)}\left(\mathcal{N}_{i}\right)}{u_{\operatorname{Max}}^{(k)}\left(\mathcal{N}_{i}\right)-u_{\min }^{(k)}\left(\mathcal{N}_{i}\right)} .
$$

3) Prioritization based on load (Load): According to this last criterion we aim at prioritizing the femtocells which currently have the highest load in their neighborhood. Hence we denote with $\ell^{(k)}\left(f_{i}\right)$ the number of users that are attached to $f_{i}$ when the radius of the cell is the one provided at iteration $k$.

We denote with $\ell_{\min }^{(k)}\left(\mathcal{N}_{i}\right)$ and $\ell_{\text {Max }}^{(k)}\left(\mathcal{N}_{i}\right)$, the minimum and the maximum of the same metric over the neighbors of $f_{i}$. Under the Load criterion of prioritization we define the value of $\alpha_{i}^{(k)}$ as follows:

$$
\alpha_{i}^{(k)}=\frac{\ell^{(k)}\left(f_{i}\right)-\ell_{\min }^{(k)}\left(\mathcal{N}_{i}\right)}{\ell_{\operatorname{Max}}^{(k)}\left(\mathcal{N}_{i}\right)-\ell_{\min }^{(k)}\left(\mathcal{N}_{i}\right)}
$$




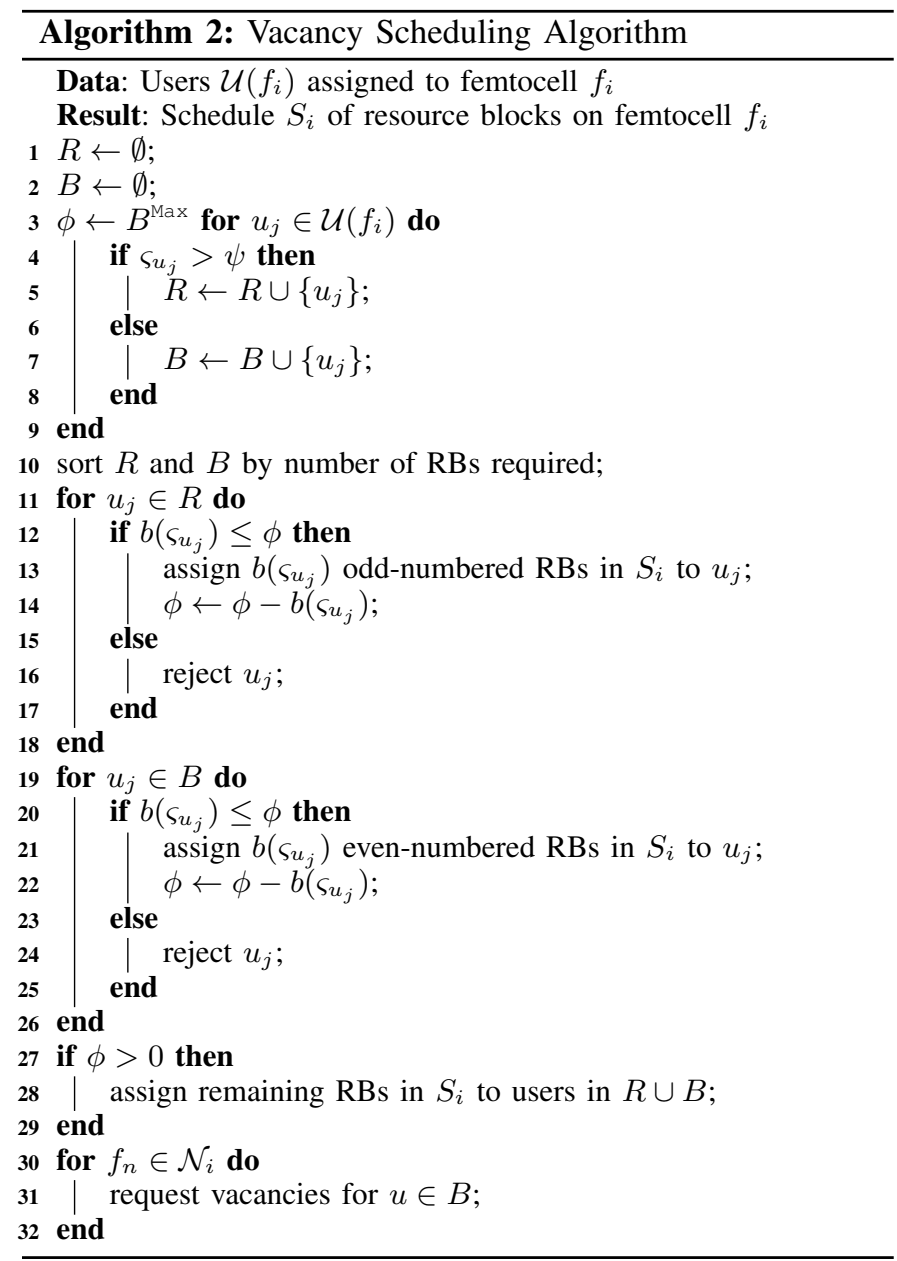

\section{Resource Block Scheduling}

In the second phase of RRS, users are scheduled onto femtocells using an interference- and throughput-aware algorithm. The algorithm divides users into two classes: regular and borderline users. Regular users are users whose measured and estimated SINRs are sufficient to meet their throughput targets. Borderline users have SINRs that are close to, but below their targets.

Regular users are scheduled first, in ascending order of the estimated number of resource blocks they require to meet their throughput targets. If a user is unable to be scheduled enough resource blocks to meet its throughput target, it is rejected. Regular users are assigned first to even-numbered resource blocks; if needed, they are scheduled on odd-numbered blocks as well.

Under all algorithm variants, borderline users are defined as users whose SINRs are within $3 \mathrm{~dB}$ below the lowest SINR threshold for service. They are scheduled after all regular users have been scheduled. Borderline users are scheduled first on odd-numbered resource blocks; they are only scheduled on even-numbered resource blocks if there is excess capacity. Borderline and regular users are scheduled in this fashion to increase the probability that borderline user vacancy requests

\begin{tabular}{c|c|c} 
Variable & Value & Description \\
\hline$L$ & $20 \log _{10}(f)+\log _{10}(d)-28 \mathrm{~dB}$ & Path loss [15] \\
$f$ & $1900 \mathrm{Mhz}$ & Carrier frequency \\
$p_{M}^{t x}$ & $40 \mathrm{dBm}$ & Macrocell tx power \\
$p_{F}^{t x}$ & $24 \mathrm{dBm}$ & Femtocell tx power \\
$\mu_{s}$ & $0 \mathrm{~dB}$ & Shadow fading mean \\
$\sigma_{s}$ & $10 \mathrm{~dB}$ & Shadow fading std dev \\
$n_{r}$ & $9 \mathrm{~dB}$ & Receiver noise figure \\
$T_{k}$ & $-174 \mathrm{dBm} / \mathrm{Hz}$ & Thermal noise density \\
$B^{\text {Max }}$ & 100 & Max RBs per base station
\end{tabular}

TABLE II: Simulation details

will be satisfied. If free resource blocks remain after all regular and borderline users have been scheduled, both regular and borderline users are scheduled on them in a round-robin fashion.

\section{A. Vacancy Requests}

Borderline users are unable to meet their throughput targets as-is. Therefore, we introduce vacancy requests, which allow a femtocell to ask its neighbors to release scheduled resource blocks. Vacancy requests consist of a list of resource block identifiers and durations. Durations are determined by the estimated number of transmission frames each user will need.

Femtocells receiving vacancy requests will only honor them on resource blocks that are assigned to users that exceed their throughput targets. This ensures that users will not fall below their throughput targets due to vacancy requests. Vacancy requests increase fairness at the expense of capacity by trading resource blocks from users that exceed their throughput targets to users that are close to meeting their throughput targets.

Since borderline users are preferentially scheduled to oddnumbered resource blocks, and regular users are preferentially scheduled to even-numbered resource blocks, if all regular users can be served at their throughput targets without spilling over to the odd-numbered resource blocks, any vacancy requests from borderline users on neighboring femtocells will be satisfied.

\section{Simulations And Results}

\section{A. Experimental Details}

The algorithms described in Section IV and V address two aspects of network operation: transmit power, via radius reduction, and resource block scheduling. Recall that the radius reduction phase can be performed according two criteria: area coverage reduction (ACR) and user coverage reduction (UCR). Additionally, recall that the radius reduction of a femtocell is performed with different priority with respect to its neighbor femtocells, according to different performance criteria, that are: estimated increase in total user throughput (TI), number of users meeting throughput requirements (UTR) and load of the femtocell (Load).

Of the six possible variants, in the following experiments, we consider the ACR-TI, the UCR-UTR, and the UCR-load variants. For each of these variants we considered both the variants for the scheduling phase, namely with or without vacancy requests. Our experiments showed that the variants UCR-TI and ACR-UTR and ACR-load performed worse than UCR-UTR, ACR-TI, and UCR-load, respectively. For the sake 


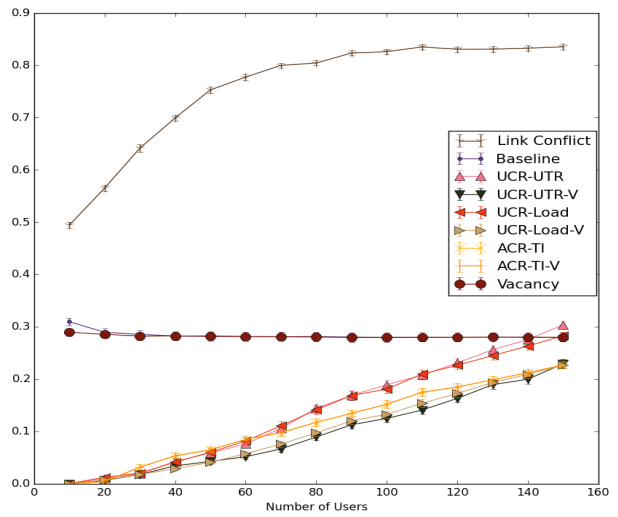

Fig. 2: Outage probability

of brevity, we omit these results. We denote with ACR-TI-V, UCR-UTR-V, UCR-load-V the variants of our algorithms in which we schedule with vacancy requests.

We evaluated a baseline algorithm that does not perform any radius reduction, and uses a naive scheduling algorithm that schedules users in a best-effort manner without vacancy requests. In the figures the baseline algorithm is referred to as Baseline. We also evaluated vacancy request scheduling on its own, with no radius reduction, indicated in the graphs as Vacancy.

To compare the performance of our algorithms to existing work, we implemented the Interfering Link Conflict algorithm, in the figures referred to as Link Conflict, from [6]. This algorithm uses graph coloring to produce mutually exclusive resource block schedules, while allowing unused resource blocks to be assigned if they meet certain interference requirements. The original paper describes a system with binary interference, where interference during a transmission results in zero throughput. By contrast, our simulated system is an SINR-based system, where interference reduces signal quality, but does not necessarily cause transmission failure. Our results show that the coloring approach of the link conflict algorithm is unsuited for SINR-based systems.

These algorithms were evaluated in a simulation of an OFDMA network modeled using LTE design principles. Details of the simulation parameters are in Table II. Users and femtocells are uniformly distributed across a $50 \mathrm{~m} \times 50 \mathrm{~m}$ area of interest.

To quantitatively measure fairness, we use Jain's index [9], defined as $f(\mathbf{X})=\frac{\left[\sum_{j=1}^{|\mathcal{U}|} x_{j}\right]^{2}}{|\mathcal{U}| \sum_{j=1}^{|\mathcal{U}|} x_{j}^{2}}$, where $0 \leq f(\mathbf{X}) \leq 1$, and $x_{j}=\frac{t_{j}}{\sum_{j=1}^{\mid \mathcal{U}} t_{j}}$. Jain's index is a commonly used measure of fairness, with $f(\mathbf{X})=1$ when each user gets a $\frac{1}{|\mathcal{U}|}$ share of total throughput, and $f(\mathbf{X})=\frac{1}{|\mathcal{U}|}$ when one users gets all throughput. A higher value for $f(\mathbf{X})$ indicates a more fair throughput distribution in the network.

\section{B. Results}

Figure 2 shows the network outage probability, defined as the percentage of users who are unable to reach their throughput targets, as the number of concurrent users increases. We considered a network composed of 30 femtocells,

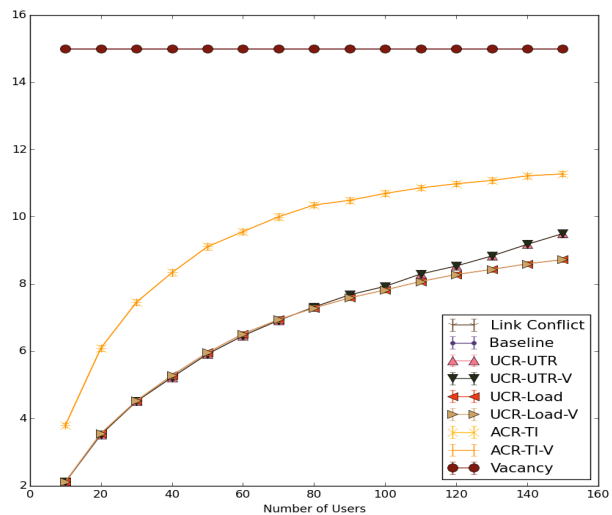

Fig. 3: Femtocell transmit power level

where user throughput requirements are distributed uniformly random between $10 \mathrm{kbps}$ and $50 \mathrm{kbps}$.

The Baseline algorithm exhibits uniform performance as the number of users increases, indicating that the network is underloaded. However, due to interference, edge users are unable to meet their throughput targets. UCR-UTR-V performs the best, closely followed by UCR-Load-V. The versions of the algorithms that do not use vacancy scheduling have higher outage probabilities, but vacancy scheduling on its own does not significantly reduce outage probabilities over the baseline.

Vacancy scheduling benefits users at cell boundaries the most, since they experience the most interference from other cells. However, when femtocell transmit powers are fixed, the reduction in interference from vacancy scheduling is typically not enough to increase an unserved user's throughput over its throughput minimum requirement. It is the combined reduction in interference due to both reduced transmit powers and vacancy scheduling that increases throughput enough for more users to meet their throughput requirements.

The Link Conflict algorithm results in outage rates that are unacceptably high. Since the algorithm eliminates conflicting transmissions between neighboring femtocells, it leaves a large number of resource blocks unassigned to prevent interference. In a system where conflicts cause transmission failure, this leads to near-optimal performance, however, in systems that can tolerate co-channel interference, the Link Conflict algorithm severely underutilizes available resources.

Figure 3 shows femtocell power levels as the number of users increases. UCR-UTR and UCR-Load consistently use less power than ACR-TI, resulting in smaller cells and lower interference. The vacancy variants decrease outage probabilities without consuming more power, but give up some resource block usage for reduced interference. This tends to reduce the throughput of high-throughput users while increasing the throughput of lower throughput users who are unable to reach their minimum throughput without assistance. The Baseline, Link Conflict, and Vacancy algorithms all use a constant amount of power as the number of users increase.

Figure 4 shows the total sum of throughput across all users. Initially, the Link Conflict and Baseline algorithms result in the highest global throughput, although the Link Conflict algorithm's global throughput falls quickly as the number 


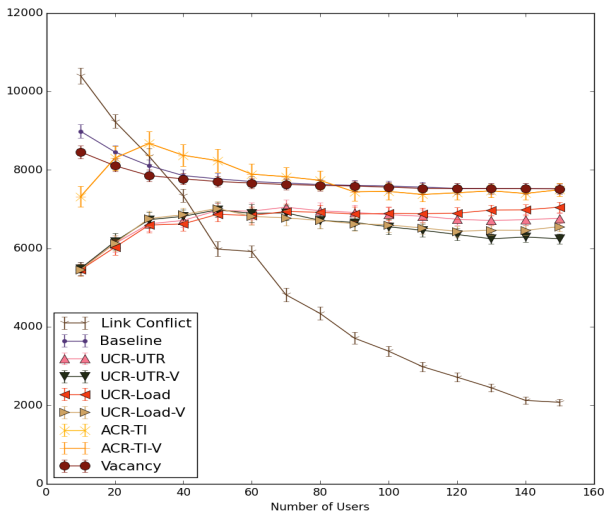

Fig. 4: Global throughput

of users increases. As the number of users increases, the throughput of the ACR-TI increases over the baseline. The ACR-TI algorithms always use less power than the baseline, indicating that the ACR-TI algorithms are able to reduce interference and increase efficiency. The UCR-UTR and UCRLoad algorithms decrease global throughput with respect to the baseline, but do so while increasing the number of users who meet their throughput targets.

The Jain's index of each algorithm, a measure of fairness, is shown in Figure 5. Recall that a Jain's index of 1 indicates a perfectly uniform throughput distribution, with each user being allocated $\frac{1}{|\mathcal{U}|}$ of the throughput, and a Jain's index of $\frac{1}{|\mathcal{U}|}$ indicates a completely unfair throughput distribution, with one user being allocated all of the throughput. The UCR-Vacancy algorithms exhibit a U-shaped Jain's index curve, with fairness increasing again as the number of users increases. The increase in fairness at high load is due to the increased use of vacancy scheduling in the UCR variants as transmission powers increase. Vacancy has a higher Jain's index than Baseline, despite serving the same number of users, indicating a fairer throughput distribution. Due to its overall poor performance, the Link Conflict algorithm has a very low fairness index, as most users get no service at all.

Overall, the UCR-Load-V and UCR-UTR-V algorithms are able to trade off some total throughput to increase the number of users that are able to connect to the network.

\section{CONCLUSION}

In this paper we presented RRS, a distributed algorithm for network management. RRS consists of two parts: an algorithm for increasing fairness in dense femtocell networks by managing femtocell transmission power using a Voronoi-Laguerre geometry-based cell radius reduction, and a scheduling algorithm that allows femtocells to request vacancies on resource blocks that are experiencing heavy interference. Simulations show that RRS reduces outage probabilities by up to $50 \%$, and increases Jain's index, a measure of fairness, by up to $190 \%$.

\section{ACKNOWLEDGMENTS}

The work of Michael Lin is supported by the William Leonhard Chair at the Pennsylvania State University, with partial support from NSF grant CNS

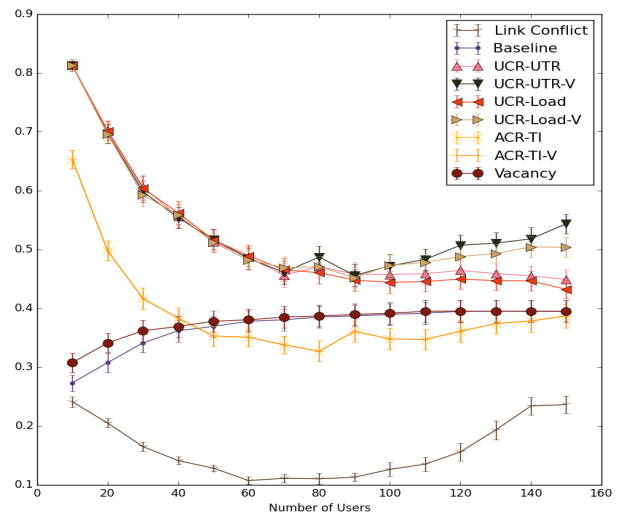

Fig. 5: Jain's index

1218597. The work of Novella Bartolini is partially supported by NATO under the SPS grant G4936 SONiCS.

\section{REFERENCES}

[1] M. Arslan, J. Yoon, K. Sundaresan, S. Krishnamurthy, and S. Banerjee, "A resource management system for interference mitigation in enterprise ofdma femtocells," Networking, IEEE/ACM Transactions on, vol. 21, no. 5, pp. 1447-1460, Oct 2013.

[2] Y. Wang, J. Zhang, J. Wang, and B. Bensaou, "Coordinated fair resource sharing in dense indoor wireless networks," in Networking Conference, 2014 IFIP, June 2014, pp. 1-9.

[3] T. Novlan, J. Andrews, I. Sohn, R. Ganti, and A. Ghosh, "Comparison of fractional frequency reuse approaches in the ofdma cellular downlink," in Global Telecommunications Conference (GLOBECOM 2010), 2010 IEEE, Dec 2010, pp. 1-5.

[4] M. Pischella and J.-C. Belfiore, "Power control in distributed cooperative ofdma cellular networks," Wireless Communications, IEEE Transactions on, vol. 7, no. 5, pp. 1900-1906, May 2008.

[5] H. Fujii and H. Yoshino, "Theoretical capacity and outage rate of ofdma cellular system with fractional frequency reuse," in Vehicular Technology Conference, 2008. VTC Spring 2008. IEEE, May 2008, pp. 1676-1680.

[6] Z. Lu, T. Bansal, and P. Sinha, "Achieving user-level fairness in openaccess femtocell-based architecture," Mobile Computing, IEEE Transactions on, vol. 12, no. 10, pp. 1943-1954, Oct 2013.

[7] J. Garcia-Morales, G. Femenias, and F. Riera-Palou, "Analytical performance evaluation of ofdma-based heterogeneous cellular networks using ffr," in Vehicular Technology Conference (VTC Spring), 2015 IEEE 81st, May 2015, pp. 1-7.

[8] H. Shi, R. Prasad, E. Onur, and I. Niemegeers, "Fairness in wireless networks:issues, measures and challenges," Communications Surveys Tutorials, IEEE, vol. 16, no. 1, pp. 5-24, First 2014.

[9] R. Jain, D. Chiu, and W. Hawe, "A quantitative measure of fairness and discrimination for resource allocation in shared systems," Digital Equipment Corporation, Tech. Rep. DEC-TR-301, 1984.

[10] F. Baccelli and B. Blaszczyszyn, "On a coverage process ranging from the boolean model to the poisson voronoi tessellation with applications to wireless communications," INRIA, Tech. Rep. RR-4019, 2000.

[11] H. Imai, M. Iri, and K. Murota., "Voronoi diagram in the Laguerre geometry and its applications," SIAM J. Comput., vol. 14, no. 1, pp. 93-105, 1985.

[12] N. Bartolini, T. Calamoneri, T. La Porta, and S. Silvestri, "Autonomous deployment of heterogeneous mobile sensors," IEEE Trans. on Mobile Computing, vol. 10, no. 6, 2011.

[13] Z. Zhou, S. Das, and H. Gupta, "Variable radii connected sensor cover in sensor networks," ACM Trans. on Sensor Networks, vol. 5, no. 1, 2009.

[14] N. Bartolini, T. Calamoneri, T. La Porta, C. Petrioli, and S. Silvestri, "Sensor activation and radius adaptation (sara) in heterogeneous sensor networks," ACM Trans. on Sensor Networks, vol. 8, no. 3, 2012.

[15] ITU, "Recommendation itu-r p.1238-4," International Telecommunication Union, Recommendation ITU-R P.1238-4, 2007. 